Artigo Original

Original Article

Weluma Evelyn Rodrigues Moura' ${ }^{1}$ (C) Camila Dalbosco Gadenz' ${ }^{1}$ Isadora de Oliveira Lemos ${ }^{1}$ (B) Ângelo José Gonçalves Bós² Mauriceia Cassol $^{1}$ (1)

Descritores

Qualidade Vocal Canto

Envelhecimento Qualidade de Vida

Keywords

Voice

Voice Quality Singing Aged Quality of Life

Endereço para correspondência: Camila Dalbosco Gadenz Departamento de Fonoaudiologia, Universidade Federal de Ciências da Saúde de Porto Alegre - UFCSPA Avenida Sarmento Leite, 245, Porto Alegre (RS), Brasil, CEP: 90050-170. E-mail: camiladalbosco@live.com

Recebido em: Outubro 27, 2020

Aceito em: Abril 07, 2021

\section{Análise do índice de desvantagem vocal para o canto de coristas idosos}

\author{
Analysis of the singing voice handicap index \\ for elderly choristers
}

\section{RESUMO}

Objetivo: Analisar o índice de desvantagem vocal para o canto de coristas idosos e verificar a sua relação com perfil, hábitos e condições de saúde dos participantes. Método: Foram incluídos 110 indivíduos com 60 anos ou mais, participantes de coros amadores. Os coristas foram entrevistados a fim de verificar dados como idade, tempo de canto coral, classificação vocal e a presença de hábitos e condições de saúde adversas à produção vocal. Posteriormente, responderam o questionário "Índice de Desvantagem Vocal para o Canto (IDV-C)" que avalia a autopercepção do indivíduo em relação às experiências no uso da voz cantada. Resultados: $\mathrm{O}$ escore do IDV-C teve uma mediana de 25 , sendo a pontuação mínima 0 e a máxima 86 . Os itens mais pontuados estavam relacionados aos aspectos físicos no uso da voz cantada: "Não consigo cantar agudo" (Q10) e "Minha garganta fica seca quando canto" (Q13). Verificou-se que os idosos com mais de 75 anos apresentaram maior desvantagem vocal para o canto ao comparar com os mais jovens $(\mathrm{p}=0,020)$. As coristas classificadas como contralto também apresentaram maior escore do IDV-C ( $\mathrm{p}=0,023)$, assim como os indivíduos que relataram beber pouca água $(\mathrm{p}=0,007)$. Conclusão: Os coristas deste estudo apresentaram um índice de desvantagem vocal para o canto compatível com vozes saudáveis para o canto. Ao verificar a relação do escore do IDV-C com as características dos participantes e a respeito dos hábitos e condições de saúde, constatou-se que os coristas idosos com mais de 75 anos, as coristas contralto e aqueles que afirmaram beber pouca água apresentaram escores maiores de desvantagem vocal para o canto.

\begin{abstract}
Purpose: To analyze the singing voice handicap index in elderly choristers and verify its relationship with the profile, habits and health conditions of the participants. Methods: 110 individuals aged 60 years or older, participating in amateur choirs, were included. Choir singers were interviewed in order to verify data such as age, time in choir singing, vocal classification, and the presence of habits and health conditions adverse to voice production. Subsequently, they answered the questionnaire "Singing Voice Handicap Index (SVHI)", which assesses the individual's self-perception in relation to experiences in the use of the singing voice. Results: The SVHI score had a median of 25 , with a minimum score of 0 and a maximum score of 86 . The most scored items were related to physical aspects in the use of singing voice: "I am unable to use my "high voice"" (Q10) and "My throat is dry when I sing"(Q13). It was found that older adults over 75 years of age had a greater voice handicap when compared to younger ones $(\mathrm{p}=0.020)$. Choir singers classified as contralto also had a higher SVHI score $(\mathrm{p}=0.023)$, as well as individuals who reported drinking little water $(\mathrm{p}=0.007)$. Conclusion: The choristers in this study presented a singing voice handicap index compatible with healthy singing voices. When verifying the relationship of the SVHI score with the characteristics of the participants and with respect to habits and health conditions, it was found that the elderly choir singers over 75 years old, the contralto choir singers, and those who claimed to drink little water had higher scores for the singing voice handicap.
\end{abstract}

Trabalho realizado na Universidade Federal de Ciências da Saúde de Porto Alegre - UFCSPA e Pontifícia Universidade Católica do Rio Grande do Sul - PUCRS - Porto Alegre (RS), Brasil.

${ }^{1}$ Departamento de Fonoaudiologia, Universidade Federal de Ciências da Saúde - UFCSPA - Porto Alegre (RS), Brasil.

${ }^{2}$ Departamento de Medicina, Pontifícia Universidade Católica do Rio Grande do Sul - PUCRS - Porto Alegre (RS), Brasil.

Fonte de financiamento: nada a declarar

Conflito de interesses: nada a declarar. 


\section{INTRODUÇÃO}

As alterações da voz podem surgir em qualquer idade por motivos diversos, porém, no decorrer do envelhecimento há um processo natural e gradual da perda da eficiência vocal conhecido como presbifonia, tendo como sintomas comuns, a presença de soprosidade, rouquidão, alterações de loudness, o aumento do pitch para os homens e diminuição para as mulheres, instabilidade e o maior esforço durante a fonação ${ }^{(1-3)}$.

As alterações vocais decorrentes do envelhecimento apresentam-se, no entanto, mais sutis em indivíduos que cantam, uma vez que o canto frequente atua como um moderador dos efeitos negativos relacionados a esse processo ${ }^{(4)}$. Ao comparar as vozes de idosos cantores e não cantores, os estudos demonstram que o primeiro grupo apresenta menos alterações de qualidade vocal, maior extensão vocal e tempos máximos de fonação mais longos ${ }^{(5-7)}$.

A prática do canto coral apresenta muitos benefícios físicos e psicossociais ao indivíduo idoso, mas também exige que os sistemas envolvidos com a fonação consigam suprir a demanda do uso vocal para o canto para que evitem gerar sobrecargas ao aparelho fonador, compensações musculares e o aumento do risco de ocorrer lesões laríngeas ${ }^{(8-10)}$. O envelhecimento é um processo natural e mesmo em idosos com um estilo de vida saudável e vocalmente ativos, as modificações anatômicas, fisiológicas e funcionais ao longo do tempo, podem gerar dificuldades e desvantagens vocais ${ }^{(7)}$.

Além disso, a falta de conhecimento e técnica contribuem para o uso inapropriado da voz e para o agravamento dos sinais e sintomas relacionados à voz. Os cantores apresentam frequentemente ressecamento do trato vocal, fadiga, desconforto, dor, sensação de aperto na garganta, tensão, quebras vocais e perda da voz, impactando a qualidade de vida destes indivíduos ${ }^{(11,12)}$.

Portanto, a identificação de situações que geram desvantagens vocais para o canto e de fatores relacionados permite que seja realizada uma atuação fonoaudiológica preventiva por meio de orientações mais efetivas, que por sua vez potencializem os efeitos do canto para a saúde vocal a esta população ${ }^{(2,3)}$. $\mathrm{O}$ presente estudo buscou analisar o índice de desvantagem vocal para o canto de coristas idosos e verificar a sua relação com perfil, hábitos e condições de saúde dos participantes.

\section{MÉTODO}

Trata-se de um estudo transversal prospectivo, aprovado pelo Comitê de Ética em Pesquisa, sob o número 1.981.615. Foram contatados nove coros amadores de uma cidade da região sul do país e sob a autorização do responsável por cada um deles, o projeto de pesquisa foi apresentado durante o ensaio dos grupos. Os coristas interessados foram entrevistados e convidados individualmente a participar da pesquisa por meio da assinatura do Termo de Consentimento Livre Esclarecido (TCLE). Foram incluídos os indivíduos com idade igual ou maior que 60 anos e que participavam do coro por pelo menos um ano. Foram excluídos da pesquisa os indivíduos tabagistas e aqueles que declararam ter realizado acompanhamento fonoaudiológico anterior ou simultâneo ao estudo.
Os idosos foram convidados a responderem o questionário intitulado "Índice Desvantagem Vocal para o Canto" (IDV-C), elaborado e validado por Cohen $^{(13)}$ e traduzido por Behlau e Gasparini ${ }^{(14)}$. O IDV-C é composto por 36 questões relacionadas com o impacto nos aspectos físico, emocional, social e econômico dos problemas vocais no canto. Para cada item, há cinco opções de respostas "Nunca", "Quase nunca", "Às vezes", "Quase sempre" e Sempre, pontuadas de 0 a 4, respectivamente. O escore total pode variar de 0 a 144 , sendo considerado o valor mínimo, o menor índice de desvantagem vocal e o valor máximo, o maior índice de desvantagem vocal. A escala não possui domínios e subescalas. Os coristas deveriam responder o questionário individualmente antes ou após o ensaio no tempo necessário para cada um e caso tivessem dúvidas em relação à alguma questão, poderiam solicitar auxílio aos pesquisadores. Foi verificado quais foram os itens do questionário mais ou menos pontuados.

Foram coletados, por meio de entrevista, os seguintes dados: idade, sexo, escolaridade, classificação vocal e tempo de canto coral. A classificação vocal já havia sido determinada anteriormente pelo regente de cada coro. Os coristas foram questionados também a respeito dos seus hábitos e condições de saúde. Foram realizadas perguntas sobre a prática de atividade física, consumo de água, uso frequente e prolongado da voz, dificuldade auditiva, alergias respiratórias, refluxo gastroesofágico, pigarro, consumo excessivo de bebidas com cafeína, bebidas destiladas e sono, totalizando dez questões. Cada pergunta poderia ser respondida com "sim" ou "não", recebendo um ponto a para cada resposta desfavorável à saúde geral e vocal, podendo somar 10 pontos. A coleta foi realizada no período de abril de 2017 a outubro de 2018.

Foi utilizado o Statistical Package for the Social Sciences (SPSS) versão 19.0 para configuração do banco de dados e análise estatística. Foram realizados os testes de KolmogorovSmirnov para a analisar a normalidade de variância. As variáveis contínuas foram descritas por meio da mediana e intervalo interquartil e as categóricas por meio da frequência absoluta e relativa. Foi utilizado o teste de U de Mann-Whitney para comparar o escore total do IDV-C entre as variáveis idade, sexo e para cada um dos hábitos e condições de saúde. $\mathrm{O}$ teste de Kruskal-Wallis foi utilizado para comparar o escore total do IDV-C entre os níveis de escolaridade, classificação vocal e tempo de canto coral. Verificou-se também a correlação de Spearman entre o escore total do IDV-C e a idade, tempo de canto coral e pontuação relacionada aos hábitos e condições de saúde. Foram considerados estatisticamente significativos os valores de $\mathrm{p}<0,05$.

\section{RESULTADOS}

Foram entrevistados 115 coristas. Destes, cinco foram excluídos: uma por ser fumante e quatro por terem realizado tratamento fonoaudiológico prévio.

A amostra teve um total de 110 coristas, com predomínio do sexo feminino $(74,5 \%)$. A faixa etária variou entre 60 a 91 anos de idade, tendo como mediana 69 (10). A maioria dos idosos apresentavam até 75 anos de idade (77,3\%). O grau de 
escolaridade de ensino superior e/ou pós-graduação foi o mais predominante $(69,1 \%)$ seguido do ensino médio $(24,5 \%)$. $O$ tempo de canto coral predominante foi maior que 10 anos $(44,5 \%)$. $\mathrm{Na}$ composição da amostra, os coristas foram classificados como soprano $(39,1 \%)$, contralto $(33,6 \%)$, tenor $(18,2 \%)$ e baixo $(9,1 \%)$.

O índice de desvantagem vocal para o canto teve uma mediana de 25 (25) sendo a pontuação mínima 0 e a máxima 86 . Conforme o Quadro 1, os itens mais pontuados estavam relacionados aos aspectos físicos no uso da voz cantada: "Não consigo cantar agudo" (item 10), "Minha garganta fica seca quando canto" (item 13), "Tenho dificuldade em cantar em forte intensidade" (item 21), "Tenho dificuldade em fazer o que quero com a minha voz" (item 17), "Quando eu canto, tenho dificuldade em controlar o ar na voz (soprosidade) (item 19), "Faço esforço para cantar" (item 1) e "Minha habilidade para cantar varia de um dia para

Quadro 1. Frequência absoluta e relativa dos itens do Índice de Desvantagem Vocal para o Canto

\begin{tabular}{|c|c|c|c|c|c|}
\hline EXPERIÊNCIAS COM A VOZ CANTADA & NUNCA & QUASE NUNCA & ÀS VEZES & QUASE SEMPRE & SEMPRE \\
\hline 1. Faço esforço para cantar & $35(31,8 \%)$ & $25(22,7 \%)$ & $41(37,3 \%)$ & $6(5,5 \%)$ & $3(2,7 \%)$ \\
\hline 2. Minha voz quebra & $32(29,1 \%)$ & $35(31,8 \%)$ & $38(4,5 \%)$ & $5(4,5 \%)$ & 0 \\
\hline 3. Estou frustrado com o meu canto & $59(53,6 \%)$ & $25(22,7 \%)$ & $21(19,1 \%)$ & $5(4,5 \%)$ & 0 \\
\hline $\begin{array}{l}\text { 4. Quando canto, as pessoas perguntam o que há de } \\
\text { errado com a minha voz }\end{array}$ & $87(79,1 \%)$ & $20(18,2 \%)$ & $1(0,9 \%)$ & $2(1,8 \%)$ & 0 \\
\hline $\begin{array}{l}\text { 5. Minha habilidade para cantar varia de um dia para } \\
\text { o outro }\end{array}$ & $33(30,0 \%)$ & $27(24,5 \%)$ & $45(40,9 \%)$ & $4(3,6 \%)$ & $1(0,9 \%)$ \\
\hline 6. Vou perdendo a voz enquanto canto & $42(38,2 \%)$ & $39(35,5 \%)$ & $24(21,8 \%)$ & $4(3,6 \%)$ & $1(0,9 \%)$ \\
\hline $\begin{array}{l}\text { 7. Fico chateado pelos problemas que a minha voz } \\
\text { cantada tem }\end{array}$ & $60(54,5 \%)$ & $27(24,5 \%)$ & $15(13,6 \%)$ & $6(5,5 \%)$ & $2(1,8 \%)$ \\
\hline $\begin{array}{l}\text { 8. Eu não quero me apresentar ou cantar por causa da } \\
\text { minha dificuldade no canto }\end{array}$ & $97(87,3 \%)$ & $10(9,1 \%)$ & $2(1,8 \%)$ & $2(1,8 \%)$ & 0 \\
\hline 9. Fico com vergonha da minha voz cantada & $76(69,1 \%)$ & $16(14,5 \%)$ & $15(13,6 \%)$ & $3(2,7 \%)$ & 0 \\
\hline 10. Não consigo cantar agudo & $28(25,5 \%)$ & $26(23,3 \%)$ & $35(31,8 \%)$ & $14(12,7 \%)$ & $7(6,4 \%)$ \\
\hline $\begin{array}{l}\text { 11. Fico nervoso antes de cantar por causa do meu } \\
\text { problema de voz }\end{array}$ & $85(77,3 \%)$ & $11(10 \%)$ & $11(10 \%)$ & $2(1,8 \%)$ & $1(0,9 \%)$ \\
\hline 12. Minha voz falada está alterada & $49(44,5 \%)$ & $34(29,1 \%)$ & $28(25,5 \%)$ & $1(0,9 \%)$ & 0 \\
\hline 13. Minha garganta fica seca quando canto & $22(20,0 \%)$ & $28(25,5 \%)$ & $50(45,5 \%)$ & $8(7,3 \%)$ & $2(1,8 \%)$ \\
\hline 14. Tive que tirar algumas músicas do meu repertório & $88(80,0 \%)$ & $17(15,5 \%)$ & $3(2,7 \%)$ & $2(1,8 \%)$ & 0 \\
\hline 15. Não confio em minha voz cantada & $52(47,3 \%)$ & $26(23,6 \%)$ & $21(19,1 \%)$ & $9(8,2 \%)$ & $2(1,8 \%)$ \\
\hline 16. Minha voz cantada está alterada & $47(42,7 \%)$ & $29(26,4 \%)$ & $30(27,3 \%)$ & $3(2,7 \%)$ & $1(0,9 \%)$ \\
\hline $\begin{array}{l}\text { 17. Tenho dificuldade em fazer o que quero com a } \\
\text { minha voz }\end{array}$ & $30(27,3 \%)$ & $34(30,9 \%)$ & $33(30,0 \%)$ & $11(10 \%)$ & $2(1,8 \%)$ \\
\hline $\begin{array}{l}\text { 18. Tenho que fazer força para encaixar a voz para } \\
\text { cantar }\end{array}$ & $44(40,0 \%)$ & $28(25,0 \%)$ & $33(30,0 \%)$ & $4(3,6 \%)$ & $1(0,9 \%)$ \\
\hline $\begin{array}{l}\text { 19. Quando eu canto, tenho dificuldade para } \\
\text { controlar o ar na voz (soprosidade) }\end{array}$ & $33(30,0 \%)$ & $26(23,6 \%)$ & $41(37,3 \%)$ & $8(7,3 \%)$ & $2(1,8 \%)$ \\
\hline $\begin{array}{l}\text { 20. Quando eu canto, tenho dificuldade de controlar a } \\
\text { aspereza na minha voz }\end{array}$ & $44(40 \%)$ & $34(30,9 \%)$ & $24(21,8 \%)$ & $8(7,3 \%)$ & 0 \\
\hline 21. Tenho dificuldade em cantar em forte intensidade & $36(32,7 \%)$ & $21(19,1 \%)$ & $39(35,5 \%)$ & $10(9,1 \%)$ & $4(3,6 \%)$ \\
\hline 22. Tenho dificuldade para manter o tom quando canto & $41(37,3 \%)$ & $33(30,0 \%)$ & $27(24,5 \%)$ & $8(7,3 \%)$ & $1(0,9 \%)$ \\
\hline $\begin{array}{l}\text { 23. Sinto-me ansioso por causa do problema em minha } \\
\text { voz cantada }\end{array}$ & $64(58,2 \%)$ & $24(21,8 \%)$ & $19(17,3 \%)$ & $2(1,8 \%)$ & $1(0,9 \%)$ \\
\hline 24. Meu canto soa forçado & $53(48,2 \%)$ & $30(27,3 \%)$ & $23(20,9 \%)$ & $4(3,6 \%)$ & 0 \\
\hline 25. Minha voz falada fica rouca depois que canto & $64(58,2 \%)$ & $25(22,7 \%)$ & $18(16,4 \% 0$ & $3(2,7 \%)$ & 0 \\
\hline 26. A qualidade da minha voz é instável & $37(33,6 \%)$ & $40(36,4 \%)$ & $25(22,7 \%)$ & $6(5,5 \%)$ & $2(1,8 \%)$ \\
\hline 27. O público tem dificuldade para me ouvir cantando & $79(71,8 \%)$ & $16(14,5 \%)$ & $9(8,2 \%)$ & $5(4,5 \%)$ & $1(0,9 \%)$ \\
\hline $\begin{array}{l}\text { 28. Sinto-me prejudicado por causa do meu problema } \\
\text { de canto }\end{array}$ & $88(80,0 \%)$ & $10(9,1 \%)$ & $8(7,3 \%)$ & $2(1,8 \%)$ & $2(1,8 \%)$ \\
\hline 29. Canso-me com facilidade quando canto & $60(54,5 \%)$ & $27(24,5 \%)$ & $21(19,1 \%)$ & $2(1,8 \%)$ & 0 \\
\hline 30. Tenho dor, coceira ou engasgos quando canto & $73(66,4 \%)$ & $24(21,8 \%)$ & $12(10,9 \%)$ & $1(0,9 \%)$ & 0 \\
\hline $\begin{array}{l}\text { 31. Quando canto, não tenho certeza de como a minha } \\
\text { voz vai sair }\end{array}$ & $52(47,3 \%)$ & $30(27,3 \%)$ & $24(21,8 \%)$ & $3(2,7 \%)$ & $1(0,9 \%)$ \\
\hline $\begin{array}{l}\text { 32. Sinto-me como se faltasse alguma coisa em minha } \\
\text { vida por causa da dificuldade que tenho de cantar }\end{array}$ & $89(80,9 \%)$ & $9(8,2 \%)$ & $9(8,2 \%)$ & $3(2,7 \%)$ & 0 \\
\hline $\begin{array}{l}\text { 33. Preocupo-me com o fato de perder dinheiro por } \\
\text { causa do meu problema de canto }\end{array}$ & $107(97,3 \%)$ & $3(2,7 \%)$ & 0 & 0 & 0 \\
\hline $\begin{array}{l}\text { 34. Sinto-me fora do cenário musical por causa do meu } \\
\text { problema de voz }\end{array}$ & $95(86,4)$ & $7(6,4 \%)$ & $6(5,5 \%)$ & $1(0,9 \%)$ & $1(0,9 \%)$ \\
\hline $\begin{array}{l}\text { 35. A dificuldade de cantar faz com que eu me sinta } \\
\text { incompetente }\end{array}$ & $91(82,7 \%)$ & $10(9,1 \%)$ & $6(5,5 \%)$ & $2(1,8 \%)$ & $1(0,9 \%)$ \\
\hline $\begin{array}{l}\text { 36. Tenho que cancelar apresentações, ensaios ou } \\
\text { compromissos de canto por causa do meu problema } \\
\text { de voz }\end{array}$ & $94(85,5 \%)$ & $14(12,75)$ & $1(0,9 \%)$ & $1(0,9 \%)$ & 0 \\
\hline
\end{tabular}


o outro" (item 5). E os menos pontuados estavam relacionados aos aspectos econômicos, sociais e emocionais: "Preocupo-me com o fato de perder dinheiro por causa do meu problema de voz" (item 33), "Tenho que cancelar apresentações, ensaios ou compromissos de canto por causa do meu problema de voz" (item 36), "Eu não quero mais me apresentar ou cantar por causa de minha dificuldade no canto" (item 8), "Sinto-me fora do cenário musical por causa do meu problema de voz" (item 34), "Quando canto, as pessoas perguntam o que há de errado com a minha voz" (item 4), "Tive que retirar algumas músicas do meu repertório" (item 14) e "A dificuldade de cantar faz com que eu me sinta incompetente" (item 35).
Os valores do questionário de acordo com as características dos participantes podem ser observados na Tabela 1. Os valores do questionário de acordo com as características dos participantes podem ser observados na Tabela 1. Ao comparar as medianas das classificações vocais femininas foi verificado que as coristas contralto apresentaram o escore de desvantagem vocal para o canto significativamente maior que as coristas soprano, enquanto não houve diferença ao comparar as classificações vocais masculinas.

Quanto aos hábitos e condições de saúde, 23 (20,9\%) relataram não praticar nenhum tipo de atividade física e 75 (68,2\%) afirmaram consumir pouca água. Além disso, $70(63,6 \%)$ informaram que falam em grande quantidade, 52 (47,3\%) que dormiam pouco,

Tabela 1. Índice de Desvantagem Vocal para o Canto de acordo com as características dos coristas

\begin{tabular}{|c|c|c|}
\hline & IDV-C $(n=110)$ & \multirow{2}{*}{ Valor $p$} \\
\hline & Mediana (IIQ) & \\
\hline Idade & & $0,020^{*}$ \\
\hline 60 a 75 & $22(23)$ & \\
\hline 76 ou mais & $32(23)$ & \\
\hline Sexo & & 0,708 \\
\hline Masculino & $20(26)$ & \\
\hline Até o Fundamental completo & $16(24)$ & \\
\hline Até o Ensino Médio completo & $24(30)$ & \\
\hline Ensino Superior e Pós-graduação & $25,50(25)$ & \\
\hline \multicolumn{3}{|l|}{ Classificação vocal } \\
\hline Baixo & $16,50(18)$ & $\mathrm{p}=0,243$ \\
\hline Tempo de canto coral & & 0,264 \\
\hline Até três anos & $22(25)$ & \\
\hline Quatro a 10 anos & $30,54(20)$ & \\
\hline Mais de 10 anos & $21(25)$ & \\
\hline
\end{tabular}

Teste Kruskal Wallis; Teste U de Mann-Whitney

${ }^{*} \mathrm{p} \leq 0,05$ valores estatisticamente significativos

Tabela 2. Relação entre os escore do IDV-C e hábitos e condições de saúde

\begin{tabular}{|c|c|c|c|}
\hline \multirow{3}{*}{ Hábitos e condições de saúde } & \multicolumn{2}{|c|}{ IDV-C $(n=110)$} & \multirow{3}{*}{ Valor $p$} \\
\hline & \multicolumn{2}{|c|}{ Mediana (IIQ) } & \\
\hline & $\operatorname{Sim}$ & Não & \\
\hline Pratica atividade física & $23(25)$ & $33(22)$ & 0,094 \\
\hline Bebe pouca água & $27(24)$ & $19(26)$ & $0,007^{*}$ \\
\hline Fala em grande quantidade & $25,50(33)$ & $22,50(33)$ & 0,458 \\
\hline Dorme pouco & $25(19)$ & $23,50(30)$ & 0,353 \\
\hline Cafeína em excesso & $26(19)$ & $23(29)$ & 0,409 \\
\hline Bebidas destiladas & $27(24)$ & $24(27)$ & 0,396 \\
\hline Dificuldade auditiva & $27(24)$ & $22(26)$ & 0,115 \\
\hline Alergias respiratórias & $25(22)$ & $24(29)$ & 0,760 \\
\hline Refluxo gastroesofágico & $26,50(22)$ & $23,50(27)$ & 0,335 \\
\hline Pigarro & $29,50(24)$ & $23,50(26)$ & 0,381 \\
\hline
\end{tabular}

Teste U de Mann-Whitney;

${ }^{*} p \leq 0,05$ valores estatisticamente significativos

Legenda: $॥ \mathrm{Q}=$ Intervalo Interquartil 
Tabela 3. Correlação entre o Índice de Desvantagem Vocal para o Canto e a idade, tempo de canto coral e pontuação do questionário de hábitos

\begin{tabular}{lcc}
\hline \multirow{2}{*}{ Variáveis } & \multicolumn{2}{c}{ Escore total IDV-C } \\
\cline { 2 - 3 } & $\mathrm{r}$ & $\mathrm{p}$ \\
\hline Idade & 0,224 & $0,021^{\star}$ \\
Tempo de canto coral & 0,100 & 0,297 \\
Pontuação de hábitos & 0,250 & $0,008^{\star}$ \\
\hline
\end{tabular}

Correlação de Spearman

${ }^{*} p \leq 0,05$ valores estatisticamente significativos

$33(30 \%)$ que consumiam bebidas com cafeína em excesso e $22(20 \%)$ bebidas destiladas. Foi identificado também que $37(33,6 \%)$ apresentavam dificuldade auditiva, $47(42,7 \%)$ alergias respiratórias, $32(29,1 \%)$ refluxo gastroesofágico e $36(32,7 \%)$ pigarro. A relação entre a mediana do escore do IDV-C para cada resposta a respeito dos hábitos e condições de saúde, representada pelo valor p, estão descritas na Tabela 2.

Houve correlações positivas, embora consideradas fracas, entre o escore total do IDV-C e a variável idade, bem como para a pontuação do questionário de hábitos e condições de saúde como pode ser observada na Tabela 3.

\section{DISCUSSÃO}

Os coristas deste estudo apresentaram um escore de IDV-C compatível com vozes saudáveis para o canto, como encontrado por Cohen (2007) na validação do instrumento utilizado ${ }^{(13)}$. O escore também foi semelhante ao valor normativo médio de um grupo de 729 cantores profissionais saudáveis com idade entre 18 e 64 anos, publicado em uma revisão sistemática recente, que apresentaram um IDV-C de 20,35 com intervalo de confiança entre 10.6 e $30.1^{(15)}$. Estudos que validaram o protocolo em diversos idiomas demonstraram que é um método válido e confiável na identificação da autopercepção do indivíduo em relação ao impacto dos problemas vocais no canto ${ }^{(16-21)}$.

Os itens relacionados a problemas físicos/funcionais foram mais pontuados do que os relacionados aos aspectos sociais/ emocionais, resultados que podem ser comparados aos de um estudo que avaliou 526 coristas amadores e constatou a presença de sintomas vocais, mas com um índice leve de ansiedade ${ }^{(9)}$. Este estudo utilizou o questionário "Índice de Desvantagem para o Canto Moderno" (IDCM) e questionários específicos para os sintomas vocais e para avaliar a ansiedade. O IDCM, assim como o IDVC, é adaptado para a voz cantada, mas este especificamente para o canto moderno. É dividido em três subescalas: incapacidade, desvantagem e defeito, que correspondem respectivamente aos domínios funcional, emocional e orgânico, o que possibilita identificar qual o domínio que o cantor apresenta maior desvantagem para o canto. O IDVC, apesar de abordar os referidos domínios em suas questões, não as mensura separadamente, ou seja, não apresenta subescalas. Entretanto, quando foi verificado quais questões obtiveram maior frequência, observou-se que aquelas relacionadas aos aspectos físicos/funcionais foram mais frequentes comparadas às demais.

Um estudo que utilizou o IDV-C para avaliar 171 cantores verificou que a idade é um fator preditivo importante para a maior desvantagem no uso da voz cantada ${ }^{(22)}$, relação verificada no presente estudo e que constatou que o escore do IDV-C dos coristas foi significativamente maior nos idosos com mais de 75 anos comparado aos mais jovens. Estes resultados sugerem que o aumento da idade contribui para a maior ocorrência de problemas no uso da voz cantada, especialmente aqueles relacionados aos aspectos físicos ${ }^{(4,13,22)}$.

Por outro lado, o hábito frequente de cantar apresenta um efeito moderador do processo de envelhecimento vocal. Um estudo que avaliou 72 adultos saudáveis verificou que o canto auxilia na manutenção da estabilidade de pitch e de amplitude, dois fatores importantes para a efetividade da comunicação. Os autores sugerem ainda que o canto, representando um treino muscular, ajudaria a manter a força e controle sobre a estabilidade vocal mesmo na presença das alterações decorrentes da idade ${ }^{(4)}$.

A prática do canto quando orientada mostra-se ser um fator de proteção da qualidade vocal tanto para cantores profissionais, quanto amadores ${ }^{(23)}$. Embora este estudo não tenha evidenciado a correlação do tempo de canto coral ao menor índice de desvantagem vocal, outra pesquisa destacou a associação inversamente proporcional nos escores de três protocolos de canto aplicados com o período de desenvolvimento da voz cantada $^{(11)}$, evidenciando que as vozes treinadas por mais tempo podem ter menos sintomas vocais quando são bem orientadas. É de fundamental importância o trabalho fonoaudiológico para o desenvolvimento da percepção vocal e auditiva dos idoso, visto que irá proporcionar habilidades vocais necessárias para a prática do canto coral, como técnicas vocais direcionadas e aumento da tessitura vocal, observando os aspectos fisiológicos envolvidos na produção da voz do idoso corista ${ }^{(4,8,20)}$.

$\mathrm{Na}$ comparação entre as classificações vocais, o estudo mostrou que há uma diferença estatisticamente significativa do escore total do IDV-C, tendo as idosas contraltos apresentado maior desvantagem vocal para o canto do que as sopranos. Esse fato concorda com outros estudos, nos quais as coristas contralto tiveram maiores escores na desvantagem para o canto ${ }^{(24)}$ e apresentaram maior cansaço vocal ${ }^{(25)}$. No presente estudo, as perguntas do IDV-C mais pontuadas e que descrevem as principais dificuldades das coristas contralto, além do alcance às notas agudas, estão relacionadas ao controle da própria voz e da soprosidade durante o canto, o que instiga a hipótese de que estas coristas estejam sob maior efeito da presbifonia. Questiona-se, portanto, se as contraltos idosas estão alocadas nessa classificação vocal devido às características vocais individuais ou por apresentarem sinais mais evidentes do processo de envelhecimento na voz, o qual acarretaria numa qualidade vocal mais grave ${ }^{(3,26)}$.

Foi encontrada uma correlação positiva, embora fraca, entre o questionário de hábitos vocais e condições de saúde e o protocolo IDV-C, sugerindo que quanto mais hábitos nocivos e condições de saúde adversas à produção vocal o indivíduo possui, maior será a sua desvantagem vocal para o canto. Os hábitos vocais e de saúde dos coristas são fatores essenciais para o adequado funcionamento do sistema fonatório e para a performance no canto, sendo itens importantes na avaliação e intervenção fonoaudiológica destes indivíduos ${ }^{(6,27)}$. 
Os achados sugerem que o hábito de tomar água esteve correlacionado à menor desvantagem vocal para o canto, confirmando o benefício da hidratação para a função vocal observado também em outros estudos ${ }^{(28,29)}$, especialmente para a voz do idoso que canta ${ }^{(29)}$. Em outro estudo a ingestão de água também mostrou-se mais prevalente em idosas coristas do que nas não $\operatorname{coristas}^{(8)}$, denotando que a prática do canto exige um maior nível de hidratação devido à sua importância para o equilíbrio do sistema fonatório na emissão da voz cantada. Este é um hábito simples por ser acessível a maioria da população, mas que contribui consideravelmente para a diminuição das queixas vocais, uma vez que favorece a flexibilidade, vibração e lubrificação das pregas vocais e, por consequência, ajuda a manter o equilíbrio biodinâmico dos mecanismos envolvidos na produção vocal ${ }^{(30)}$.

Nesse estudo não foram observadas diferenças significativas nos escores do IDV-C associadas ao RGE, alergias respiratórias e sexo, estando em consonância com outra pesquisa relacionada a fatores associados a desvantagem para o canto ${ }^{(22)}$. No entanto para a variável sexo é possível verificar na literatura uma maior desvantagem vocal em mulheres cantoras, bem como em não cantoras relacionado, principalmente, aos aspectos funcionais do uso da voz ${ }^{(16)}$. Quanto ao RGE e alergias respiratórias, sabe-se que quando presentes estes podem prejudicar a produção vocal. Nosso estudo não detectou esses efeitos a partir do escore do IDV-C, possivelmente, porque a investigação foi realizada com base nos sintomas auto-referidos pelos participantes e não verificados através de exames objetivos ou histórico médico.

As demais variáveis sobre hábitos e condições de saúde não apresentaram relação significativa com a pontuação do IDV-C, mesmo as conhecidas adversas à voz. Provavelmente pela metodologia do questionário aplicado, que abordou questões somente de "Sim" ou "Não", não avaliando a frequência e nem duração dessas variáveis na rotina dos idosos que participaram da pesquisa.

Com relação às limitações do estudo, destacam-se a ausência de avaliação de imagem laríngea para a detecção de possíveis alterações de estruturas do trato vocal e a falta de especificidade do instrumento IDV-C para a aplicação em populações idosas. Neste sentido, sugere-se mais pesquisas que considerem a avaliação otorrinolaringológica e a construção de um instrumento que identifique características vocais e dificuldades específicas de populações de coristas idosos.

O estudo demonstrou uma mediana de 25(25) na pontuação total do protocolo, associações positivas com idade e classificação vocal, além de uma correlação positiva com hábitos vocais. Destacamos a importância do uso de instrumentos de autopercepção vocal e de questionários de saúde vocal em coros amadores, a fim de mapear possíveis dificuldades apresentadas por esta população, possibilitando uma intervenção fonoaudiológica baseada nas necessidades do coro. Em estudos futuros, sugerimos a adaptação do instrumento para a aplicação em coristas idosos, visando atender às particularidades e às demandas envolvidas na atividade do canto coral nesta fase da vida.

\section{CONCLUSÃO}

Os coristas deste estudo apresentaram um índice de desvantagem vocal para o canto compatível com vozes saudáveis para o canto. Ao verificar a relação do escore do IDV-C com as características dos participantes e a respeito dos hábitos e condições de saúde, constatou-se que os coristas idosos com mais de 75 anos, as coristas contralto e aqueles que afirmaram beber pouca água apresentaram escores maiores de desvantagem vocal para o canto.

\section{REFERÊNCIAS}

1. Çiyiltepe M, Şenkal ÖA. The ageing voice and voice therapy in geriatrics. Aging Clin Exp Res. 2017;29(3):403-10. http://dx.doi.org/10.1007/s40520016-0597-3. PMid:27324692.

2. Mezzedimi C, Di Francesco M, Livi W, Spinosi MC, De Felice C. Objective evaluation of presbyphonia: spectroacoustic study on 142 patients with Praat. J Voice. 2017;31(2):257.e25-32. http://dx.doi.org/10.1016/j. jvoice.2016.05.022. PMid:27427181.

3. Rapoport SK, Menier J, Grant N. Voice changes in the elderly. Otolaryngol Clin North Am. 2018;51(4):759-68. http://dx.doi.org/10.1016/j.otc.2018.03.012. PMid:29887345.

4. Lortie CL, Rivard J, Thibeault M, Tremblay P. The moderating effect of frequent singing on voice aging. J Voice. 2017;31(1):112.e1-12. http:// dx.doi.org/10.1016/j.jvoice.2016.02.015. PMid:27049451.

5. Maruthy S, Ravibabu P. Comparison of dysphonia severity index between younger and older carnatic classical singers and nonsingers. J Voice. 2015;29(1):65-70. http://dx.doi.org/10.1016/j.jvoice.2014.05.001. PMid:25179778.

6. Aquino FS, Silva MAA, Teles LCS, Ferreira LP. Aspects of the speaking voice of elderly women with choral singing experience. CoDAS 2016;28(4):446-53. http://dx.doi.org/10.1590/2317-1782/20162015109. PMid:27652927.

7. D'haeseleer E, Claeys S, Bettens K, Leemans L, Van Calster A-S, Van Damme $\mathrm{N}$, et al. The impact of a teaching or singing career on the female vocal quality at the mean age of 67 years: a pilot study. J Voice. 2017;31(4):516. e19-26. http://dx.doi.org/10.1016/j.jvoice.2016.12.016. PMid:28187923.

8. Cassol M, Bós AJG. Impact of choir singing in elderly on depression symptoms and voice quality. Public Health Prev Med. 2015;1(3):101-7.

9. Rosa M, Behlau M. Mapping of vocal risk in amateur choir. J Voice. 2017;31(1):118.e1-11. http://dx.doi.org/10.1016/j.jvoice.2016.03.002. PMid:27055399.

10. Bullack A, Gass C, Nater UM, Kreutz G. Psychobiological effects of choral singing on affective state, social connectedness, and stress: influences of singing activity and time course. Front Behav Neurosci. 2018;12:223. http://dx.doi.org/10.3389/fnbeh.2018.00223. PMid:30319371.

11. Murry T, Zschommler A, Prokop J. Voice handicap in singers. J Voice. 2009;23(3):376-9. http://dx.doi.org/10.1016/j.jvoice.2007.10.013. PMid: 18191378 .

12. Phyland DJ, Oates J, Greenwood KM. Self reported voice problems among three groups of professional singers. J Voice. 1999;13(4):602. http://dx.doi. org/10.1016/S0892-1997(99)80014-9. PMid:10622525.

13. Cohen SM, Jacobson BH, Garrett CG, Noordzij JP, Stewart MG, Attia A, et al. Creation and validation of the singing voice handicap index. Ann Otol Rhinol Laryngol. 2007;116(6):402-6. http://dx.doi. org/10.1177/000348940711600602. PMid:17672240.

14. Behlau M, Gasparini G. Índice de Desvantagem Vocal para o Canto - IDV-C. Tradução [Internet] 2020 [citado em 2020 Jul 13]. Disponível em: http:// www4.pucsp.br/laborvox/dicas_pesquisa/downloads/IDVC.pdf

15. Sobol M, Sielska-Badurek EM, Osuch-Wójcikiewicz E. Normative values for singing voice handicap index: systematic review and meta-analysis. Rev Bras Otorrinolaringol. 2020;86(4):497-501. http://dx.doi.org/10.1016/j. bjorl.2018.12.004. PMid:30846419.

16. Paoliello K, Oliveira G, Behlau M. Singing voice handicap mapped by different self-assessment instruments. CoDAS. 2013;25(5):463-8. http:// dx.doi.org/10.1590/S2317-17822013005000008. PMid:24408551. 
17. Castelblanco L, Habib M, Stein DJ, de Quadros A, Cohen SM, Noordzij JP. Singing voice handicap and videostrobolaryngoscopy in healthy professional singers. J Voice. 2014;28(5):608-13. http://dx.doi.org/10.1016/j. jvoice.2014.03.003. PMid:24836361.

18. Gunjawate DR, Aithal VU, Guddattu V, Bellur R. Adaptation and validation of the Kannada version of the singing voice handicap index. J Voice. 2017;31(4):507.e7-11. http://dx.doi.org/10.1016/j.jvoice.2016.10.012. PMid:27913091.

19. Baracca G, Cantarella G, Forti S, Pignataro L, Fussi F. Validation of the Italian version of the Singing Voice Handicap Index. Eur Arch Otorhinolaryngol. 2014;271(4):817-23. http://dx.doi.org/10.1007/s00405-013-2658-y. PMid:23974331.

20. Sielska-Badurek E, Osuch-Wójcikiewicz E, Sobol M, Kazanecka E, Niemczyk K. Singers' vocal function knowledge levels, sensorimotor selfawareness of vocal Tract, and Impact of functional voice rehabilitation on the vocal function knowledge and self-awareness of vocal tract. J Voice. 2017;31(1):122.e17-24. http://dx.doi.org/10.1016/j.jvoice.2016.01.011. PMid:26952318.

21. Ghaemi H, Dehqan A, Mahmoodi-Bakhtiari B, Sobhani-rad D. The Singing Voice Handicap Index (SVHI): Validation and adaptation into Persian. J Voice. 2020;34(1):158.e17-23. http://dx.doi.org/10.1016/j.jvoice.2018.07.025. PMid:30170911.

22. Cohen SM, Noordzij JP, Garrett CG, Ossoff RH. Factors associated with perception of singing voice handicap. Otolaryngol Head Neck Surg. 2008;138(4):430-4. http://dx.doi.org/10.1016/j.otohns.2007.12.041. PMid: 18359349 .

23. Prakup B. Acoustic measures of the voices of older singers and nonsingers. J Voice. 2012;26(3):341-50. http://dx.doi.org/10.1016/j.jvoice.2011.05.007. PMid:21889299.

24. Rezende G, Irineu RA, Dornelas R. College choir: self-reported symptoms vocal and handicap vocal in singing. Rev CEFAC. 2015;7(4):1161-72. http://dx.doi.org/10.1590/1982-0216201517415114.
25. Coelho ACC, Daroz IF, Silverio KCA, Brasolotto AG. Amateur choir singers: self-image, difficulties and symptoms of the singing voice. Rev CEFAC. 2013;15(2):436-43. http://dx.doi.org/10.1590/S1516-18462013000200021.

26. Siqueira LTD, Silverio KCA, Berretin-FÉlix G, Genaro KF, Fukushiro AP, Brasolotto AG. Influence of vocal and aerodynamics aspects on the voicerelated quality of life of older adults. J Appl Oral Sci. 2020;28:e20200052. http://dx.doi.org/10.1590/1678-7757-2020-0052. PMid:32813841.

27. Crawley BK, Dehom S, Thiel C, Yang J, Cragoe A, Mousselli I, et al. Assessment of clinical and social characteristics that distinguish presbylaryngis from pathologic presbyphonia in elderly individuals. JAMA Otolaryngol Head Neck Surg. 2018;144(7):566-71. http://dx.doi. org/10.1001/jamaoto.2018.0409. PMid:29799925.

28. Siqueira MA, Bastilha GR, Lima JPM, Cielo CA. Vocal hydration in voice professionals and in future voice professionals. CEFAC. 2016;18(4):90814. http://dx.doi.org/10.1590/1982-0216201618417415.

29. van Wyk L, Cloete M, Hattingh D, van der Linde J, Geertsema S. The effect of hydration on the voice quality of future professional vocal performers. J Voice. 2017;31(1):111.e29-36. http://dx.doi.org/10.1016/j. jvoice.2016.01.002. PMid:26873423.

30. Alves M, Krüger E, Pillay B, van Lierde K, van der Linde J. The effect of hydration on voice quality in adults: a systematic review. J Voice. 2019;33(1):125.e13-28. http://dx.doi.org/10.1016/j.jvoice.2017.10.001. PMid:29122414.

\section{Contribuição dos autores}

Todos os autores participaram suficientemente do trabalho para tornar pública sua responsabilidade sobre o seu conteúdo. WERM participou da coleta, análise e interpretação dos dados e redação do artigo; CDG participou da coleta, análise e interpretação dos dados e redação do artigo; IOL participou da interpretação dos dados e revisão do artigo; AJGB participou, na condição de coorientador, da idealização do estudo e revisão do artigo; MC participou, na condição de orientadora, da idealização do estudo, da interpretação dos dados e revisão do artigo. 